\title{
A Damage Detection Algorithm Utilizing Dynamic Displacement of Bridge under Moving Vehicle
}

\author{
Zhen Sun, ${ }^{1,2}$ Tomonori Nagayama, ${ }^{1}$ Di Su, ${ }^{1}$ and Yozo Fujino ${ }^{3}$ \\ ${ }^{1}$ Department of Civil Engineering, The University of Tokyo, 7-3-1 Hongo, Bunkyo-ku, Tokyo 113-8656, Japan \\ ${ }^{2}$ Jiangsu Transportation Institute, 2200 Chengxin Street, Nanjing 211112, China \\ ${ }^{3}$ Institute of Advanced Sciences, Yokohama National University, 79-1 Tokiwadai, Hodogaya-ku, Yokohama 240-8501, Japan
}

Correspondence should be addressed to Zhen Sun; sunzhen08@gmail.com

Received 30 July 2015; Revised 21 September 2015; Accepted 4 October 2015

Academic Editor: Matteo Aureli

Copyright (c) 2016 Zhen Sun et al. This is an open access article distributed under the Creative Commons Attribution License, which permits unrestricted use, distribution, and reproduction in any medium, provided the original work is properly cited.

\begin{abstract}
A damage detection method is proposed, which utilizes dynamic displacement of bridge structures under moving vehicle. The problem is first elaborated with closed-form solution of dynamic displacement, which is decomposed into quasi-static component and dynamic component. Dynamic curvature is defined as second derivative of the dynamic displacement for detecting damage location and estimating damage extent. Damage is modeled by local reduction of stiffness in this paper. Numerical study was conducted on a simply supported beam to verify the proposed method. Vehicle model is analyzed with Newmark's method using Matlab to obtain the contact force acting on the bridge. Beam model is established in commercial finite element software ABAQUS. The effects of road surface roughness and vehicle-bridge interaction are both considered in the analysis. In order to identify damage location and extent, dynamic curvature was calculated with midspan displacement. Parametric study on measurement noise level, damage location, damage extent, and multiple damage cases is performed, and the analysis results show both reliability and efficacy of this method in damage detection of bridge structures. At last, conclusions are drawn for its application to bridges in engineering practice.
\end{abstract}

\section{Introduction}

During the service life of a bridge, factors such as material aging and harsh environmental conditions as well as extreme events such as natural disasters may cause structural damage to varying degrees [1]. In order to avoid severe accident of bridge collapse, detection of any potential damage is important for serviceability and safety of bridges.

In recent years, vibration based methods have been widely studied for bridge assessment. Detailed review of the damage detection methods can be found in [2,3]. Most of these methods utilize mode frequency or mode shapes as the identification parameter. However, there are several difficulties for these frequency domain methods: (1) baseline of previous test data of undamaged structure is needed, which is often unavailable; (2) frequency, as a global modal parameter, is not sensitive to local damage; (3) frequency is often affected by environmental conditions such as temperature or humidity. These difficulties have prevented practical use of vibration based methods.

Besides the frequency domain methods, some efforts are devoted to investigating the problem with time domain damage detection methods. In the time domain methods, a moving vehicle is often used as external excitation to obtain the dynamic response of the bridge. The basic concept is that the moving vehicle will pass every section of the bridge, which includes the damage part. When the vehicle is right over the damage range, singularity will be caused in the response compared with other parts. Through data-processing techniques to find singularity in the signal, damage location could be detected. Huang [4] applied Hilbert-Huang Transform (HHT) to a model of simply supported damaged bridge under loading of a moving truck. The undamaged model is taken as baseline, and variation of local frequency and 
deformation is employed to detect damage. Some other researchers used wavelet analysis to detect singularity in bridge response signal, hence detecting damage in bridges. Zhu and Law [5] conducted wavelet analysis on bridge response under a moving load. Wavelet coefficient of dynamic displacement is obtained to detect damage in a bridge model. Nguyen and Tran [6] conducted similar study, but, instead of using bridge response, they used vehicle response for wavelet analysis, which makes it unnecessary for sensor installation on a bridge. Hester and González [7] performed wavelet transform on response of a damaged bridge under moving load and investigated feasibility to detect damage location. They concluded that multiple measurement points were required to achieve higher accuracy. In the waveletbased damage detection techniques, there is a major inherent difficulty. In order to localize damage, narrow window of bridge response, that is, short time series, is needed. However, frequency resolution is impaired when short time series are chosen to calculate the frequency. Due to this contradiction, effective detection of damage is difficult.

In this paper, a curvature based damage detection method is proposed, which uses dynamic displacement of a bridge subject to loading of a moving vehicle. It does not require baseline of undamaged state of bridge, and both damage location and damage extent can be detected. Firstly, the physical mechanism of dynamic displacement of damaged bridge under loading of a moving vehicle is elaborated. Dynamic displacement is decomposed into quasi-static component and dynamic component, and curvature is used to detect damage location and estimate damage extent. Secondly, the proposed method is verified with a finite element model using beam elements, in which vehicle model and beam model are established in Matlab and ABAQUS, respectively. Vehiclebridge interaction and roughness are both considered, and dynamic displacement is used to calculate curvature and detect damage. The effect of parameters of measurement noise level, damage location, damage extent, and multiple damage cases is studied, which illustrates reliability and efficacy of the proposed method. Finally, conclusions are made for application of this method in engineering practice.

\section{Damage Detection with Dynamic Displacement}

The dynamic problem of vehicle-bridge system has been a subject of interest for a long time. In the earlier times, most of the studies are aimed at predicting dynamic response of bridges and providing a dynamic amplification factor for engineering design [8]. Detailed review of the early analytical and numerical studies could be found in previous literature such as $[9,10]$. In the following section, physical background is elaborated, and it is shown that dynamic curvature is a good indicator for damage detection.

2.1. Analysis on Dynamic Displacement under Moving Vehicle. In simplified case of an undamaged uniform simply supported beam, vehicle is modeled as a moving load with

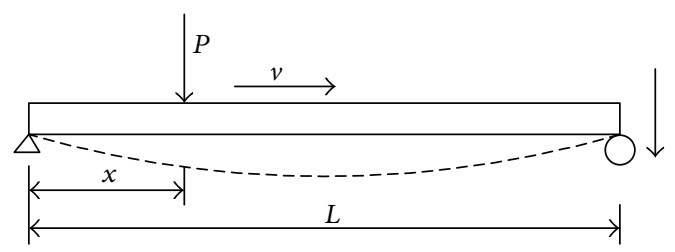

FIGURE 1: Schematic figure of simply supported beam under moving load.

constant speed $v$, as illustrated in Figure 1. The closedform solution was deduced by Weaver et al. [11], and the displacement of the bridge is expressed as follows:

$$
\begin{aligned}
y(x, t)= & -\frac{2 P L^{3}}{m \pi^{2}} \sum_{i=1}^{\infty} \frac{\sin (i \pi x / L)}{i^{2}\left(i^{2} \pi^{2} a^{2}-v^{2} L^{2}\right)} \sin \left(\frac{i \pi v t}{L}\right) \\
& +\frac{2 P L^{4} v}{m \pi^{3} a} \sum_{i=1}^{\infty} \frac{\sin (i \pi x / L)}{i^{3}\left(i^{2} \pi^{2} a^{2}-\dot{u}^{2} L^{2}\right)} \sin \omega_{n} t
\end{aligned}
$$

where $a=\sqrt{E I / m} ; \omega_{n}=\left(i^{2} \pi^{2} / L^{2}\right) \sqrt{E I / m} ; L$ is the length of the bridge; $P$ is the amplitude of the moving load; $m$ is the mass per unit length; $E I$ is the flexural rigidity of the bridge; $v$ is the velocity of the moving force; $\omega_{n}$ is the natural frequency of the bridge.

It is noticed that the first part is related to the vehicle speed, which is referred to as the quasi-static component; the second part contains bridge natural frequency and is referred to as dynamic component, which comes from the vibration of the bridge. Figure 2 illustrates the dynamic displacement at midspan, which is composed of these two parts: quasi-static component and dynamic component. The proposed method concentrates on calculating curvature from the quasi-static component of the dynamic displacement, and an innovative technique is used in curvature calculation to cancel out the dynamic displacement.

2.2. Damage Detection with Curvature. The relation between bending moment and curvature of a beam is expressed in the following equation [12]:

$$
M(x)=-E I(x) \frac{d^{2} w(x)}{d x^{2}},
$$

where $E$ denotes Young's modulus of the material, $I$ denotes the second moment of area of beam cross section, $w(x)$ denotes deflection of the beam, $x$ denotes the beam coordinate, and $M(x)$ denotes the bending moment.

Curvature can be calculated from the second derivative of deflection measurements, which is expressed as

$$
\kappa(x)=\frac{d^{2} w(x)}{d x^{2}}=-\frac{M(x)}{E I(x)} .
$$

If there is damage (stiffness loss) in some location of the beam, abrupt change is expected at the corresponding location on the curvature plot. 

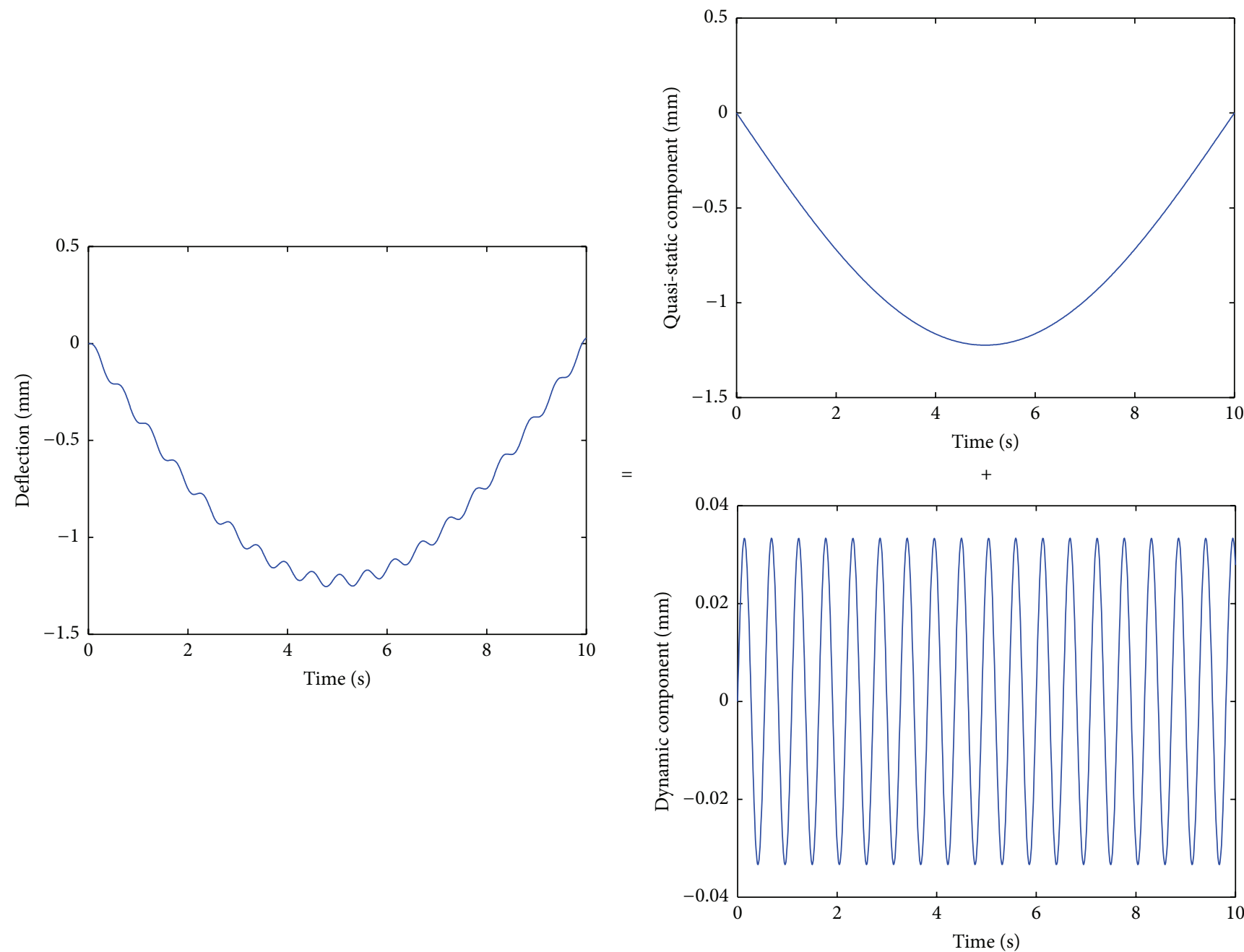

FIgURE 2: Quasi-static and dynamic components in deflection signal.

In this study, damage extent is defined as $\alpha$, which indicates loss in stiffness $E I$ :

$$
\alpha=\frac{E I_{i}-E I_{d}}{E I_{i}},
$$

where the subscripts $i$ and $d$ denote "intact" and "damaged," respectively.

The stiffness in intact and damaged states is defined, respectively, as

$$
\begin{aligned}
E I_{i} & =-\frac{M(x)}{\kappa_{i}(x)} \\
E I_{d} & =-\frac{M(x)}{\kappa_{d}(x)} .
\end{aligned}
$$

Defining $\Delta \kappa=\kappa_{d}-\kappa_{i}$ and substituting (5) and (6) into (4) yields

$$
\frac{\Delta \kappa}{\kappa_{i}}=\frac{\alpha}{1-\alpha} .
$$

Hence, damage extent can be calculated as

$$
\alpha=\frac{\Delta \kappa / \kappa_{i}}{\Delta \kappa / \kappa_{i}+1} .
$$

Curvature can be approximated with second derivative of displacement measurements:

$$
\kappa(x)=w^{\prime \prime}(x)=\frac{w(x+\Delta x)-2 w(x)+w(x-\Delta x)}{\Delta x^{2}},
$$

where $w(x)$ denotes displacement. Illustration of the proposed method is shown in Figure 3. In order to obtain dense deflection measurement, Maxwell-Betti Reciprocal Theorem is utilized and it is explained as follows. Consider a beam in two states under different loads. Maxwell-Betti Reciprocal Theorem states that the work of the forces of the first state on the displacements of the second state is equal to the work of forces of the second state on the displacements of the first state. Based on this theorem, the deflection of a beam at point $A$ under a load at point $B$ is the same as the deflection at point $\mathrm{B}$ under the same load at point $\mathrm{A}$. By extending this equivalence relationship to a moving load, the static 

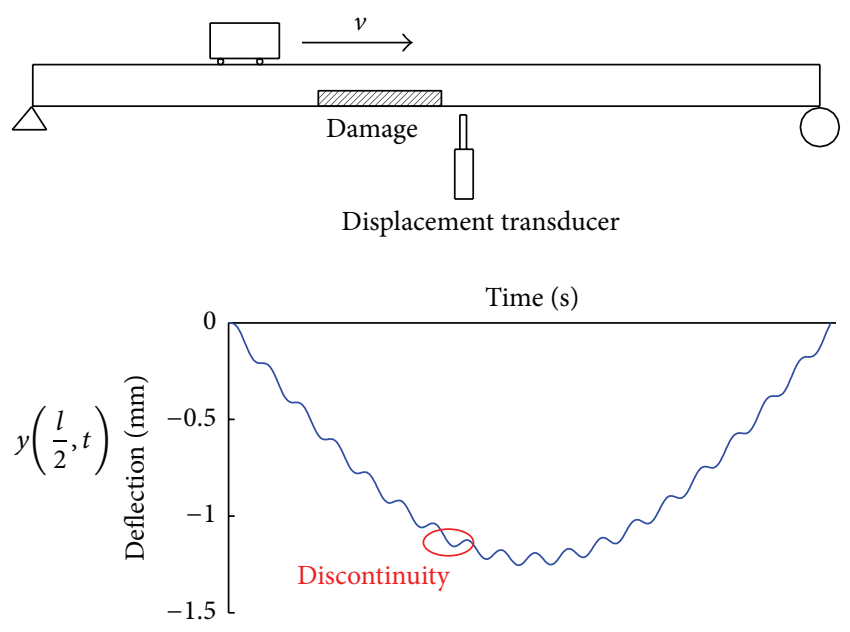

FIGURE 3: Illustration of damage identification from dynamic displacement.

deflection $w(x)$ of a beam under a load at point $\mathrm{A}, P(A)$, is obtained with deflection measurement at point $\mathrm{A}, w(A)$, under a moving load $P(x)$. The load is assumed to move slowly so that the dynamic effect is negligible. In application to engineering practice, the vehicle load is assumed to be point load, and the bridge is modeled as a beam.

For dynamic displacement of a damaged bridge under vehicle loading, it contains three parts: quasi-static component, dynamic component, and damage-induced component. Therefore (9) can be expressed as

$$
\begin{array}{r}
\kappa(x)=\frac{w_{\text {static }}(x-\Delta x)-2 w_{\text {static }}(x)+w_{\text {static }}(x+\Delta x)}{\Delta x^{2}} \\
+\frac{w_{\text {dynamic }}(x-\Delta x)-2 w_{\text {dynamic }}(x)+w_{\text {dynamic }}(x+\Delta x)}{\Delta x^{2}} \\
+\frac{w_{\text {damage }}(x-\Delta x)-2 w_{\text {damage }}(x)+w_{\text {damage }}(x+\Delta x)}{\Delta x^{2}} .
\end{array}
$$

Noticing that dynamic component is a periodic function (Figure 2), in curvature calculation formula, calculation interval $\Delta x$ can be chosen as the length of the natural period:

$$
\Delta x=T_{n} \times f_{s},
$$

where $T_{n}$ denotes fundamental natural period of the bridge; $f_{s}$ denotes sampling frequency of data acquisition. In this way, the three terms in the numerator of dynamic component are canceled out, while contribution from damage is still kept in the calculated curvature. For a beam, the fundamental natural period is integer times of higher bending mode periods. Therefore, higher bending mode components are also canceled out by the option of $\Delta x$.

In this analysis, $w(x)$ is dynamic displacement instead of static deflection, and $\Delta x$ is chosen as the length of fundamental natural period. $\kappa(x)$ is defined as dynamic curvature to distinguish with the curvature calculated with static deflection.

In this proposed method, as shown in Figure 3, a displacement transducer is installed at one position, while a

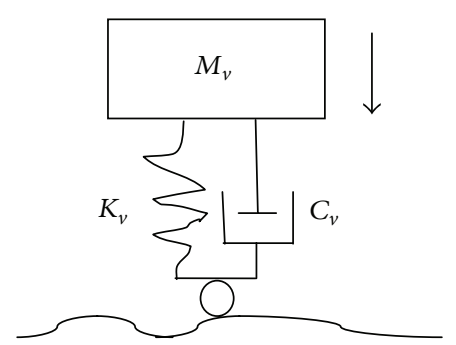

FIGURE 4: Quarter-car model.

vehicle passes through a bridge. If there is discontinuity in recorded dynamic displacement signal, it will correspond to damage on the bridge. Dynamic curvature will highlight the damage location, and damage extent can also be calculated from curvature values.

\section{Investigation with a Finite Element Model}

In order to verify the proposed damage detection method, investigation is conducted on a vehicle-bridge model using Matlab and finite element software ABAQUS. In the analysis, the vehicle model is established using Matlab, and the contact force at the wheel is calculated with Newmark's method. The bridge is modeled as a simply supported beam in ABAQUS, and contact force is imported from Matlab calculation result. The analysis scheme can be used to compute the bridge behavior with good accuracy and high efficiency.

\subsection{Numerical Example}

3.1.1. Vehicle Model. Equation of motion for the vehicle is obtained using the principle of virtual work. The vehicle loading is simulated with a quarter-car model shown in Figure 4 composed of a spring with stiffness coefficient of $8 \times 10^{3} \mathrm{~N} / \mathrm{m}$ and a damper with damping coefficient of $1.44 \times$ $10^{3} \mathrm{~N} \cdot \mathrm{s} / \mathrm{m}$. The mass of vehicle body is $100 \mathrm{~kg}$. Considering the mass of tire is much smaller than the vehicle body, it is neglected in the calculation.

The equation of motion for the vehicle is

$$
M_{v} \ddot{U}_{v}+C_{v} \dot{U}_{v}+K_{v} U_{v}=f_{v},
$$

where $M_{v}, C_{v}, K_{v}$ denote mass, damping coefficient, and stiffness of the vehicle, respectively; $\ddot{U}_{v}, \dot{U}_{v}, U_{v}$ denote acceleration, velocity, and displacement of vehicle motion, respectively; $f_{v}$ denotes the vertical force on the vehicle, which is contributed from road roughness function and bridge displacement at the contact points. Constant vehicle speed and straight vehicle path are assumed in this study. The dynamic equation is calculated in Matlab with Newmark's method, and the contact force on the bridge is calculated as follows:

$$
f=f_{v}+M_{v} g-C_{v} \dot{U}_{v}-K_{v} U_{v}
$$

3.1.2. Bridge Model. Steel beam model is established with three-dimensional beam element B31 in commercial finite 
TABLE 1: Properties of the beam model.

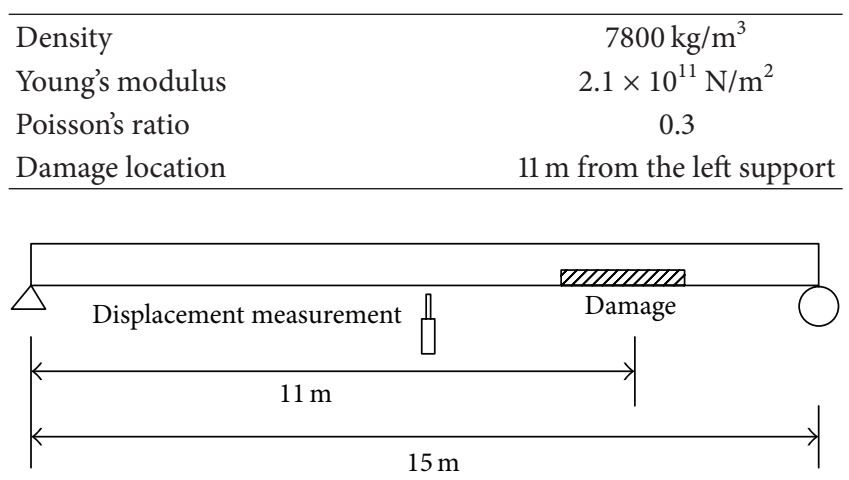

FIGURE 5: Layout of bridge model.

element software ABAQUS shown in Figure 5. It is $15 \mathrm{~m}$ long and $1 \mathrm{~m}$ wide, with a thickness of $0.06 \mathrm{~m}$, and the boundary condition is specified as simply supported. The beam is modeled totally with 200 beam elements.

The bridge specifications are provided in Table 1. Damage is simulated by reduction of cross section, which is $11 \mathrm{~m}$ away from the left end, with a length of $0.75 \mathrm{~m}$, and the thickness reduced to $0.05 \mathrm{~m}$. Damage extent is $42 \%$ stiffness loss. In analysis, vehicle passes at a constant speed of $1 \mathrm{~m} / \mathrm{s}$, so totally it takes $15 \mathrm{~s}$ to pass the bridge. Rayleigh damping is defined for the model.

Road surface is continuously distributed in a random fashion, which affects dynamic behavior of both vehicle and bridge. It is usually assumed to be a stationary Gaussian random process $[13,14]$ and is generated from power spectral density functions. Consider

$$
r(x)=\sum_{k=1}^{N} \sqrt{2 G_{d}\left(n_{k}\right) \Delta n} \cos \left(2 \pi n_{k} x-\theta_{k}\right),
$$

where $G_{d}\left(n_{k}\right)=a /\left(2 \pi n_{k}\right)^{2}$ is power spectral density function and $a$ denotes road condition and is chosen as $2 \times 10^{-6} ; \Delta n$ is the frequency interval; $\Delta n=\left(n_{\max }-n_{\min }\right) / N ; n_{\max }$ and $n_{\min }$ are the upper and lower cut-off frequencies, respectively; here they are chosen as $5 \mathrm{~Hz}$ and $0.1 \mathrm{~Hz} ; n_{k}$ is the wave number; $\theta_{k}$ is the random phase angle uniformly distributed from 0 to $2 \pi$.

When calculating the dynamic behavior of the vehicle, the space function is transformed to time function according to the vehicle speed. The generated road profile is shown in Figure 6, with the largest amplitude of roughness below $2 \mathrm{~mm}$.

3.1.3. Calculation Algorithm. In this analysis, interaction between the bridge and the vehicle is realized through contact force. At the contact points between the vehicle wheel and the bridge, displacement of the vehicle and the bridge should satisfy compatibility at the contact points, where the same deformation of both the vehicle and the bridge should be maintained. Convergence should be satisfied at every contact point.

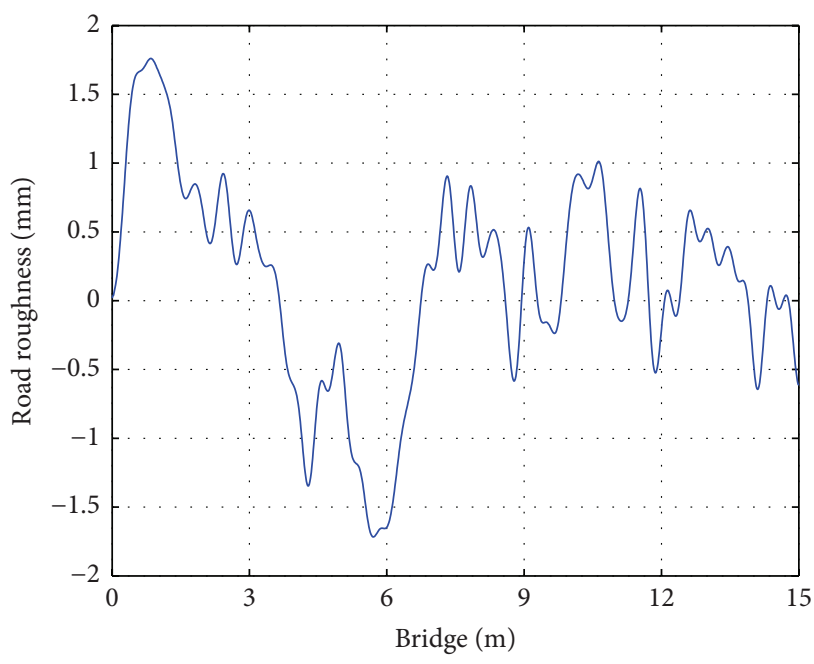

FIGURE 6: Bridge road surface profile.

There has been step by step algorithm for the computation [8], in which the vehicle moves through the bridge, and the contact force is calculated from the vehicle behavior at each contact point. Then the contact force is input to the bridge, which causes bridge response and the response is fed back to the vehicle model. After that the vehicle moves to the next point, and the process continues until the vehicle passes the bridge. In addition to step by step algorithm, there is also iterative procedure which treats the contact force as time history [15]. In this analysis, computation procedure is conducted in a global manner presented in Figure 7, and interaction between the vehicle and the bridge is realized through the contact force time history. First, road profile is used as the initial input for the vehicle, and the contact force history is calculated for each contact point. The contact force history is then applied to the bridge model in ABAQUS, which leads to bridge response. Then the bridge response is incorporated in the vehicle dynamic equation as input, together with the road profile, and a new contact force time history is generated. This procedure is repeated until user defined convergence criteria are satisfied. Particularly the convergence criterion is set that the maximum deflection difference between successive iterations is smaller than $0.01 \%$ of the dynamic displacement at midspan. Once the convergence criterion is satisfied, displacement response of the bridge is extracted for damage detection.

3.1.4. Analysis Result. Analysis is conducted until convergence is satisfied. The difference of dynamic displacement at the midspan point is shown in Figure 8. It indicates that, after three iterations, the maximum difference between two iterations is $5 \times 10^{-7} \mathrm{~m}$, around $0.0025 \%$ of the midspan deflection of the bridge. It satisfies the convergence criterion that the displacement difference is smaller than $0.01 \%$ of the midspan deflection.

Dynamic displacement at the midspan point is extracted for damage detection given in Figure 9. The natural frequencies of the bridge were also extracted, which shows that 


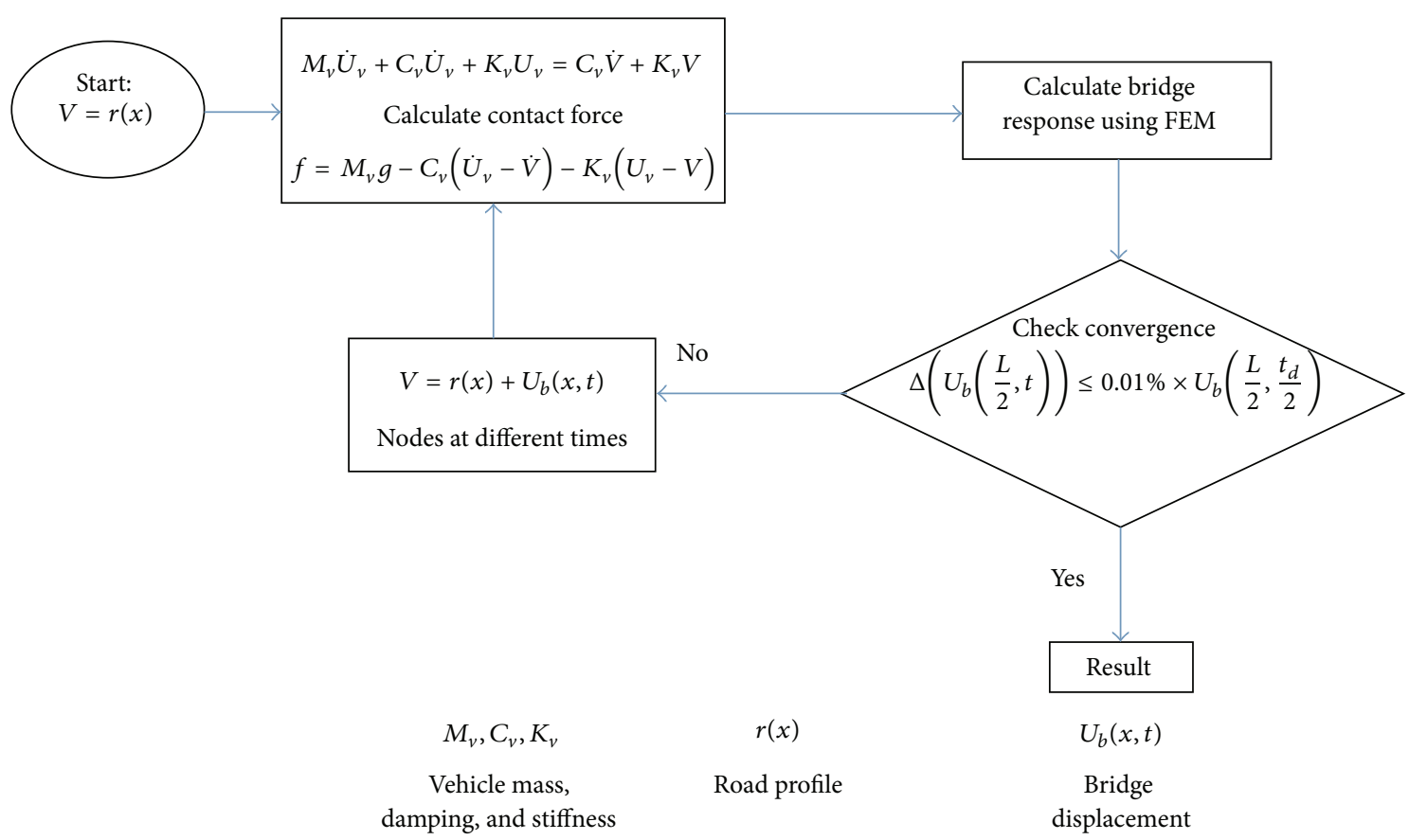

FIgURE 7: Calculation algorithm for vehicle-bridge model.

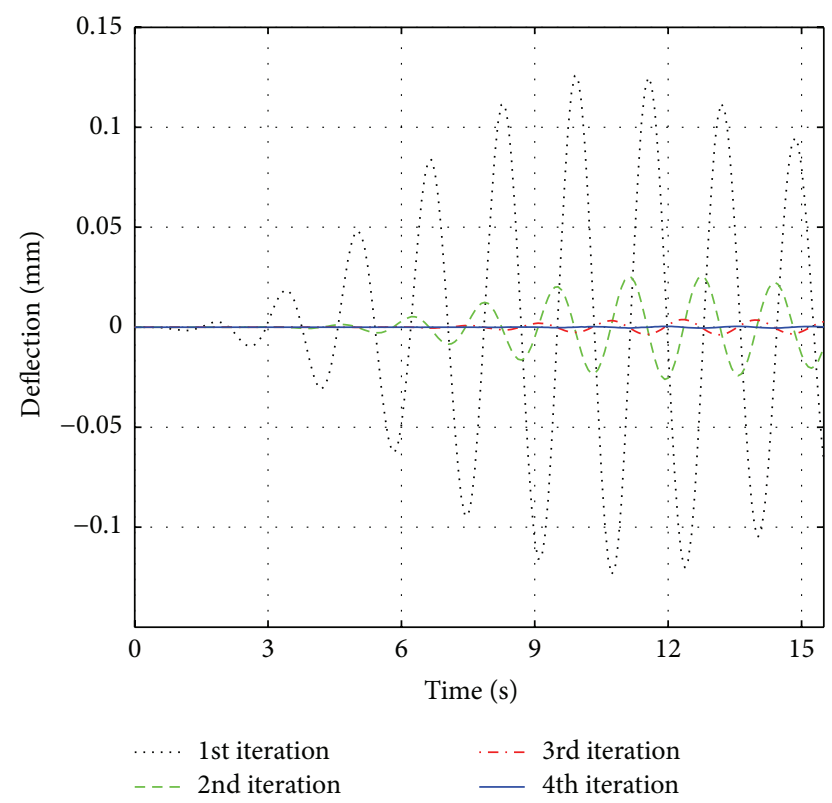

FIGURE 8: Convergence of displacement at contact points.

the fundamental natural frequency is $0.60765 \mathrm{~Hz}$. Hence, the fundamental natural period is obtained as $1.648 \mathrm{~s}$. In data processing, the second derivatives of the dynamic displacement data are taken at an interval of the natural period of the bridge. The vehicle passes the bridge in $15 \mathrm{~s}$, and displacement is recorded. Therefore, $f_{s}$ is calculated as $13.4 \mathrm{~Hz}$. For the calculation of dynamic curvature, $\Delta x$ is chosen as $\Delta x=T_{n} \times$ $f_{s} . \Delta x$ is then rounded to an integer value, which is 22 .

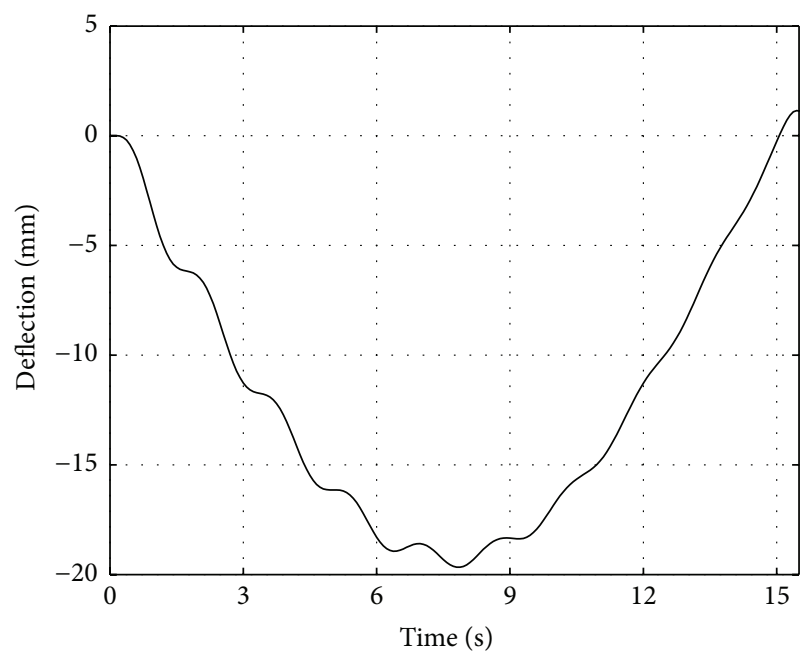

Figure 9: Dynamic displacement at the center of the bridge span.

The dynamic curvature is illustrated in Figure 10. When the vehicle-bridge interaction is not considered, the dynamic curvature is also presented for comparison, given by the red dashed line. In the curvature figure, there are no calculated curvature values at both ends of the bridge span, because the second derivative was taken at an interval $\Delta x$. It can be indicated from the figure that when interaction is not considered, curvature plot is smoother, and peak caused by damage is more apparent. In contrast, there are more fluctuations on the curvature result which incorporated interaction between the vehicle and the bridge. But, even considering the interaction effect, the damage location is clearly identified. 


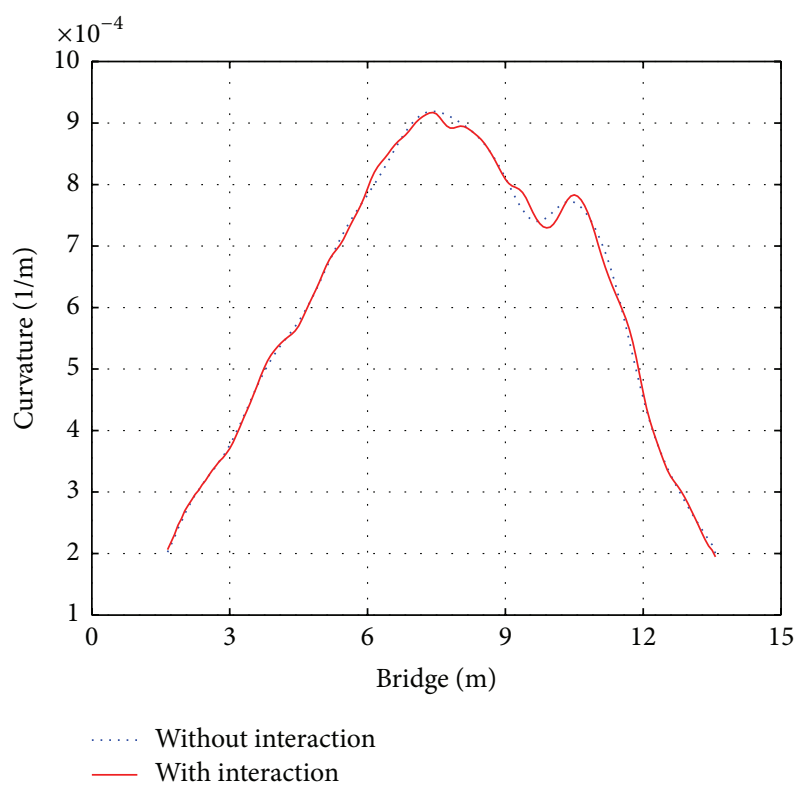

FIGURE 10: Curvature of bridge at midpoint of the span.

Apparent peak at the location of $11 \mathrm{~m}$ on the dynamic curvature plot indicates the damage location. Using the damage extent calculation equation (9), the damage extent is obtained as $39.98 \%$. Compared with the actual damage extent of $42 \%$, the difference is around $2 \%$. It demonstrates that, even with the effects from road roughness and vehicle-bridge interaction, the damage detection method can detect damage location and identify damage extent with good accuracy.

3.2. Effect of Measurement Noise. In order to investigate the effect of measurement noise on efficacy of the proposed method, random noise is generated and added to the calculated displacement response of the bridge. The displacement with measurement noise is calculated as $[5,6]$

$$
w=w_{c}+E_{p} N \sigma\left(w_{c}\right)
$$

where $w$ is the polluted bridge displacement with measurement noise. $E_{p}$ is the noise level and $N$ is a standard normal distribution with zero mean value and unit standard deviation. $w_{c}$ is the calculated displacement at midpoint of bridge span, and $\sigma\left(w_{c}\right)$ is its standard deviation.

Three different noise levels of $0.1 \%, 0.3 \%$, and $0.5 \%$ are investigated, and curvature result is shown in Figure 11. It is shown that damage location can be successfully determined for all three cases. However, damage extent is less accurate, which is identified as $31.3 \%, 30.1 \%$, and $28.2 \%$, respectively. This is due to the fact that curvature values are distorted by measurement noise. The proposed method can both successfully locate the damage and provide a quantitative estimation of the damage extent.

3.3. Effect of Damage Location and Extent. In order to investigate the effect of damage location and extent, different damage locations and extents are analyzed. Damage is

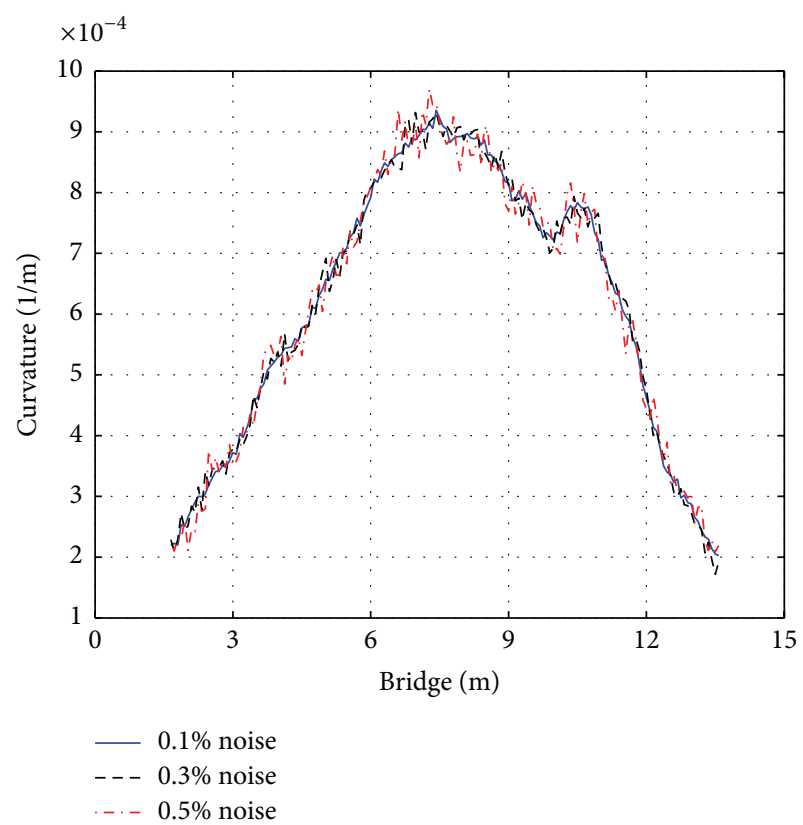

FIgURE 11: Curvature at midspan with different noise level.

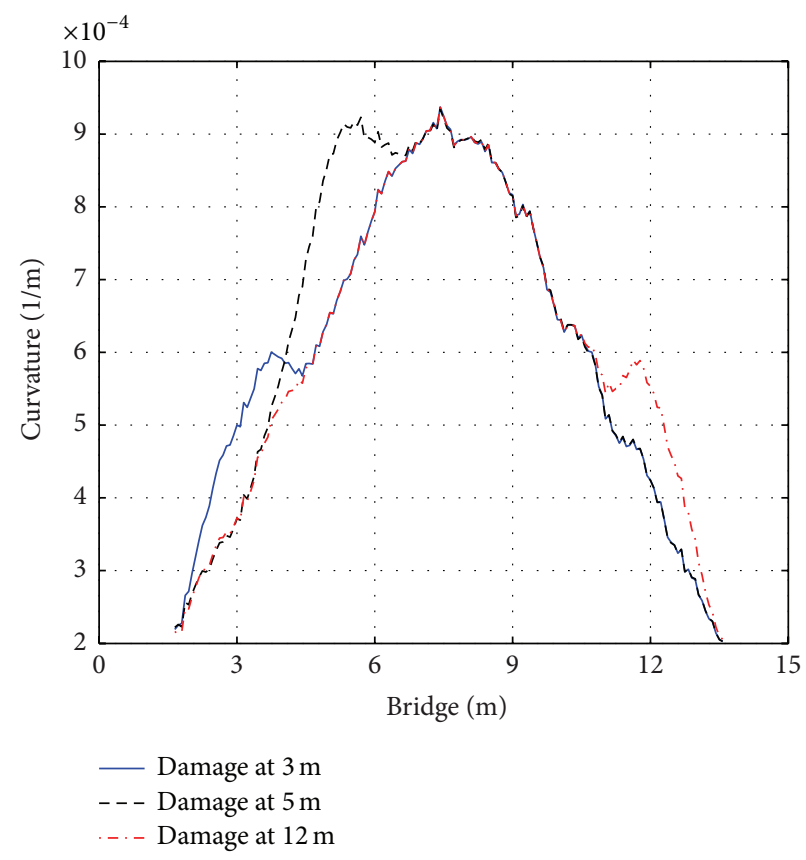

FIGURE 12: Curvature at midspan with different damage location.

assumed at location of $3 \mathrm{~m}(\mathrm{~L} / 5), 5 \mathrm{~m}(\mathrm{~L} / 3)$, and $12 \mathrm{~m}(4 \mathrm{~L} / 5)$, respectively, and damage length is $0.75 \mathrm{~m}$ with thickness reduced to $0.05 \mathrm{~m}$. Noise level is assumed as $0.1 \%$, and it is added to calculated displacement in the same way as in the last subsection. All other conditions are defined the same as in Section 3.1. The curvature results are illustrated in Figure 12. It is shown that damage can be detected reliably regardless of its location. Damage extent is identified as $36.8 \%, 32.9 \%$, and $33.9 \%$, respectively. 


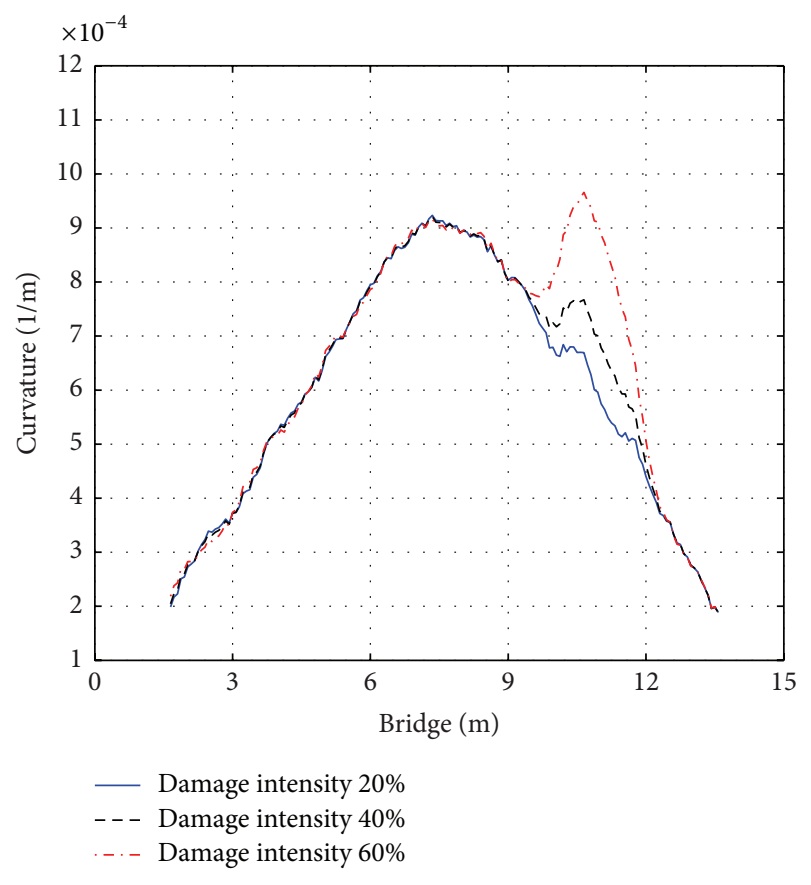

FIGURE 13: Curvature at midspan with different damage extent.

Effect of damage extent is also studied, by assuming different damage extent of $20 \%, 40 \%$, and $60 \%$. Noise level is assumed as $0.1 \%$, and all other conditions are defined the same as in Section 3.1. The curvature results are plotted in Figure 13, which shows that the peak caused by damage increases as damage extent increases from $20 \%$ to $60 \%$. Damage extent is identified as $17.9 \%, 32.2 \%$, and $49.6 \%$. The discrepancy can be attributed to the road surface roughness and measurement noise.

3.4. Effect of Multi-Damage Case. In this subsection, the beam with multiple damage is studied. The bridge model is the same as in Section 3.1 except that multiple damage cases are artificially created. Totally damage is generated at three locations, which are $5 \mathrm{~m}(1 / 3 \mathrm{~L}), 10 \mathrm{~m}(2 / 3 \mathrm{~L})$, and $12 \mathrm{~m}(4 / 5 \mathrm{~L})$ of the bridge. The damage extent is $42 \%$ for all cases, and $0.1 \%$ noise level is added to calculated displacement.

Figure 14 shows results of the curvature at midspan of the bridge. It is observed that all three damage cases can be detected at corresponding locations. Damage extent is also identified using (9), which is $33.7 \%, 34.8 \%$, and $32.3 \%$, respectively.

A finite element bridge model is used to investigate reliability and efficacy of the proposed method. Effects of measurement noise, damage location and extent, and a multidamage case are studied, which show that the proposed method can be used to detect damage location and estimate damage extent successfully. It is noted that the purpose of this paper is to verify the proposed damage detection algorithm numerically, and a simple supported beam model is adopted. Further application to real bridges should take into account high local modes and torsion modes.

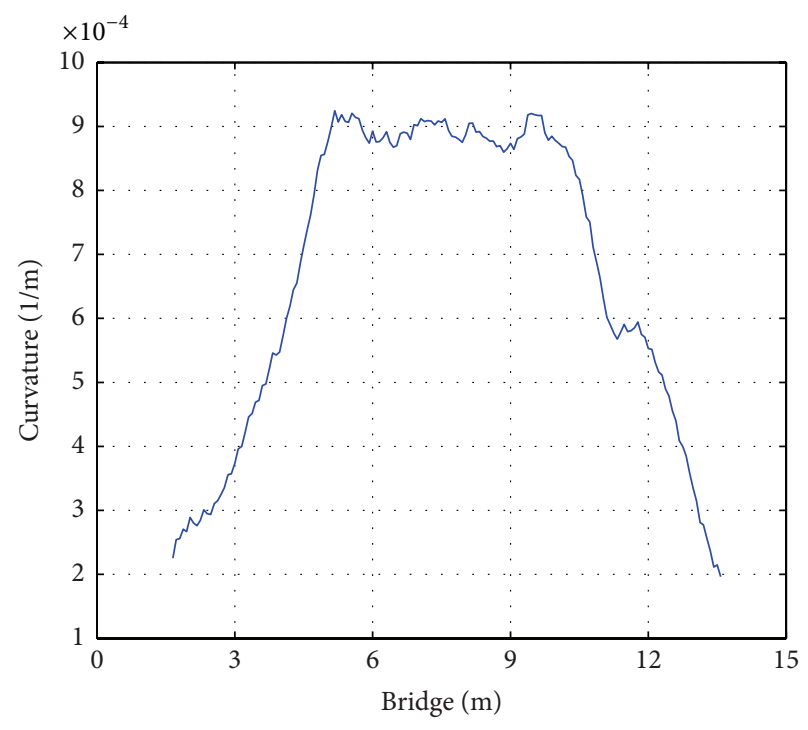

FIgURE 14: Curvature at midspan with multiple damage cases.

\section{Conclusions}

Safety of bridges during operation is of much concern for the society, which requires more reliable and efficient damage detection methods. In this paper, a damage detection method is proposed based on dynamic displacement of bridge under the loading of a moving vehicle. Through this study, several conclusions are drawn as follows:

(1) Closed-form solution of the vehicle-bridge problem shed light on decomposing dynamic displacement into quasi-static component and dynamic component, and curvature of the quasi-static component can be used to detect damage location and estimate damage extent.

(2) In curvature calculation, using dynamic displacement, the calculation interval is chosen as natural period of the beam, by which dynamic component can be canceled out, while damage is detected with quasi-static component.

(3) Numerical simulation of a beam model is investigated, in which road surface roughness and vehiclebridge interaction are considered. Parametric study on measurement noise, damage location, damage extent, and multiple damage cases is performed, which verified the reliability and efficacy of this method.

In light of damage detection of bridges, this method utilizes dynamic displacement of bridges under moving vehicle loading, which can be operated with low cost and simple implementation. Moreover, baseline from undamaged state is not required, and both damage location and damage extent can be assessed. In field test measurements of real bridges, there could be higher local modes or torsional modes, which should be considered in application of the proposed method. Further investigations will be conducted to study these issues in future. 


\section{Conflict of Interests}

The authors declare that there is no conflict of interests regarding the publication of this paper.

\section{Acknowledgments}

The authors would like to express gratitude to Professor Dionysius from Yokohama National University for his advices on this research. The first author is also grateful for the scholarship from MEXT (Ministry of Education, Culture, Sports, Science and Technology, Japan) during his Ph.D. study.

\section{References}

[1] Y. Fujino and D. M. Siringoringo, "Bridge monitoring in Japan: the needs and strategies," Structure and Infrastructure Engineering, vol. 7, no. 7-8, pp. 597-611, 2011.

[2] O. S. Salawu, "Detection of structural damage through changes in frequency: a review," Engineering Structures, vol. 19, no. 9, pp. 718-723, 1997.

[3] S. W. Doebling, C. R. Farrar, and M. B. Prime, "A summary review of vibration-based damage identification methods," The Shock and Vibration Digest, vol. 30, pp. 91-105, 1998.

[4] K. Huang, US 6192758 B1 United States, 2001.

[5] X. Q. Zhu and S. S. Law, "Wavelet-based crack identification of bridge beam from operational deflection time history," International Journal of Solids and Structures, vol. 43, no. 7-8, pp. 2299-2317, 2006.

[6] K. V. Nguyen and H. T. Tran, "Multi-cracks detection of a beam-like structure based on the on-vehicle vibration signal and wavelet analysis," Journal of Sound and Vibration, vol. 329, no. 21, pp. 4455-4465, 2010.

[7] D. Hester and A. González, "A wavelet-based damage detection algorithm based on bridge acceleration response to a vehicle," Mechanical Systems and Signal Processing, vol. 28, pp. 145-166, 2012.

[8] J.-C. Wyss, D. Su, and Y. Fujino, "Prediction of vehicle-induced local responses and application to a skewed girder bridge," Engineering Structures, vol. 33, no. 4, pp. 1088-1097, 2011.

[9] S. P. Timoshenko, History of Strength of Materials, D. Van Nostrand Company, New York, NY, USA, 1953.

[10] L. Fryba, Vibration of Solids and Structures Under Moving Loads, Academia Prague, Prague, Czech Republic, 1999.

[11] J. R. W. Weaver, S. P. Timoshenko, and D. H. Young, Vibration Problems in Engineering, John Wiley \& Sons, New York, NY, USA, 1990.

[12] S. P. Timoshenko, Strength of Materials, D. Van Nostrand Company, New York, NY, USA, 1955.

[13] C. J. Dodds and J. D. Robson, "The description of road surface roughness," Journal of Sound and Vibration, vol. 31, no. 2, pp. 175-183, 1973.

[14] Y.-B. Yang and B.-H. Lin, "Vehicle-bridge interaction analysis by dynamic condensation method," Journal of Structural Engineering, vol. 121, no. 11, pp. 1636-1643, 1995.

[15] M. David, Finite Element Analysis, Sciyo, 2010. 


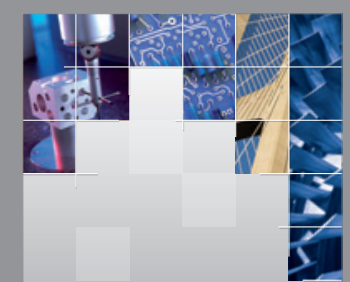

\section{Enfincering}
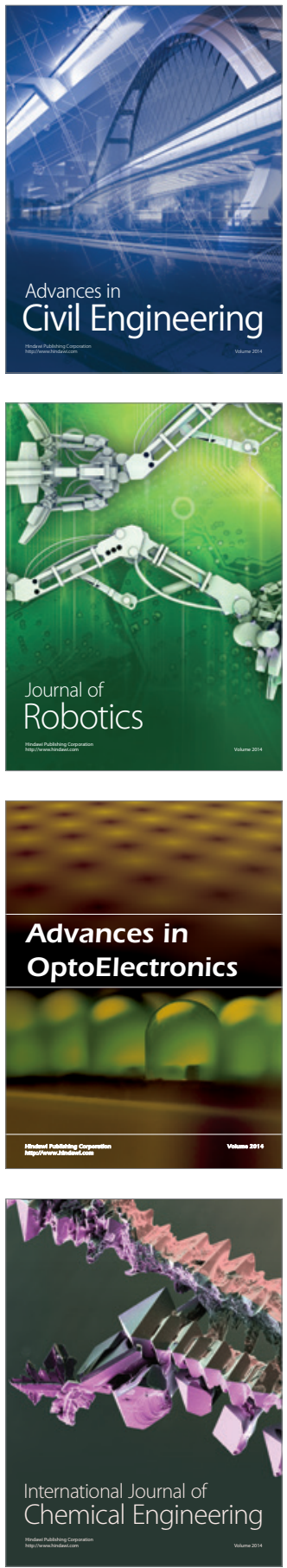

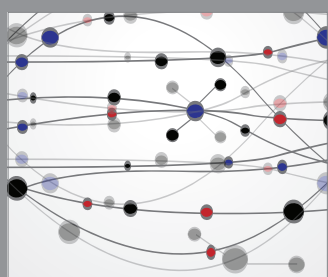

The Scientific World Journal

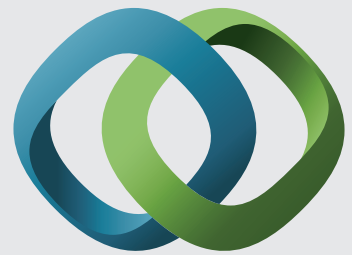

\section{Hindawi}

Submit your manuscripts at

http://www.hindawi.com
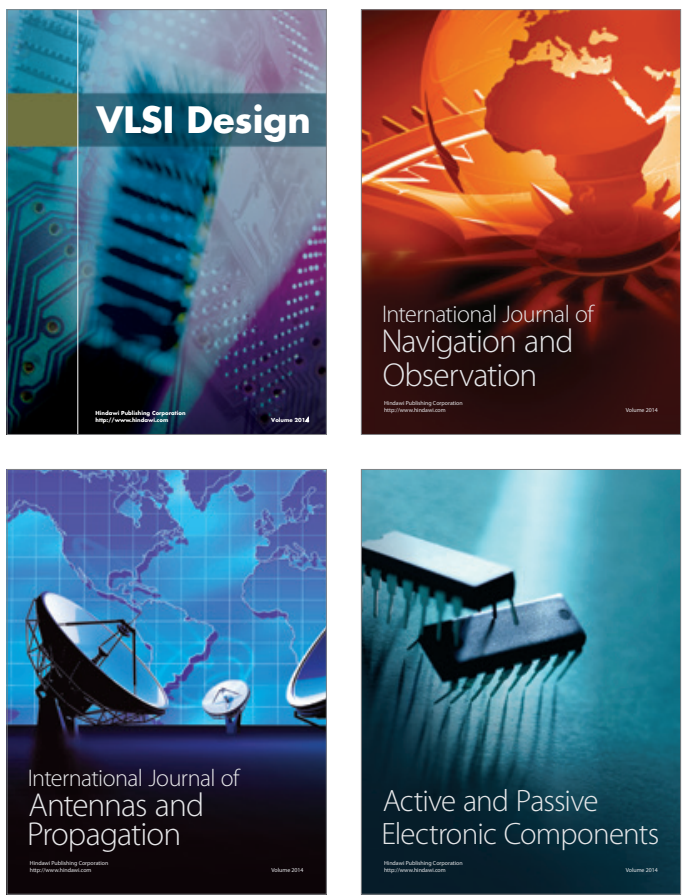
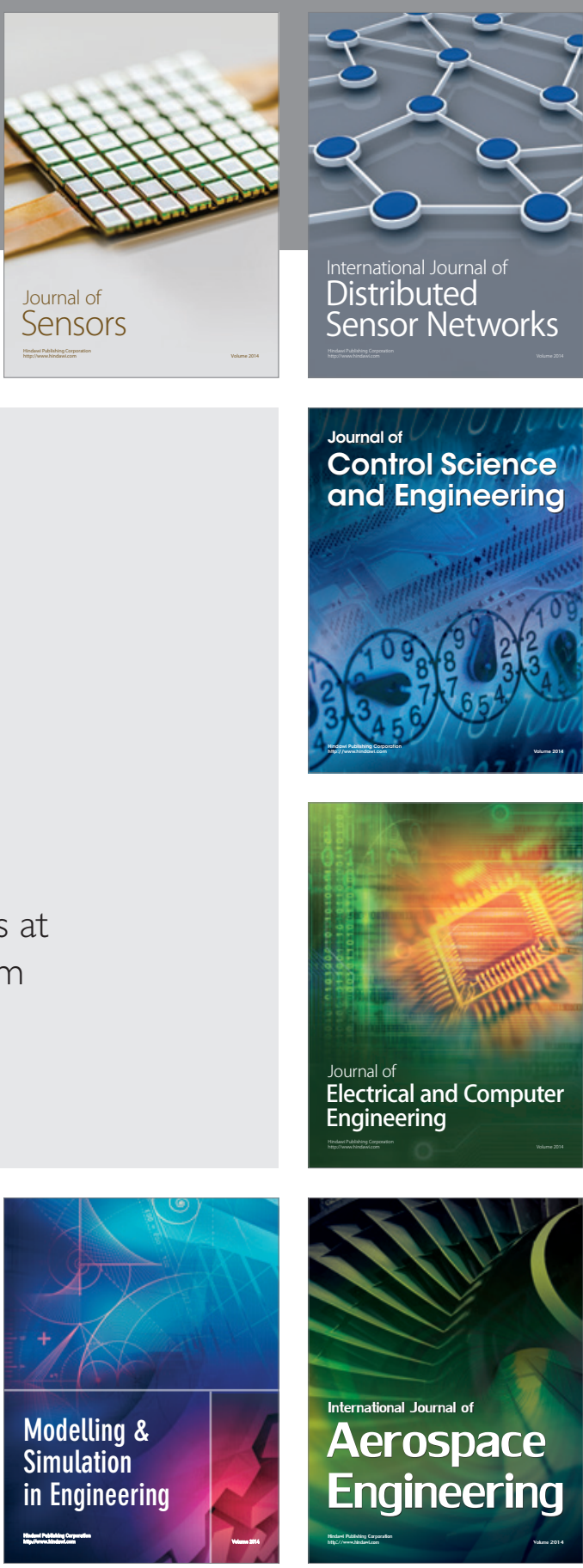

International Journal of

Distributed

Sensor Networks

Journal of

Control Science

and Engineering
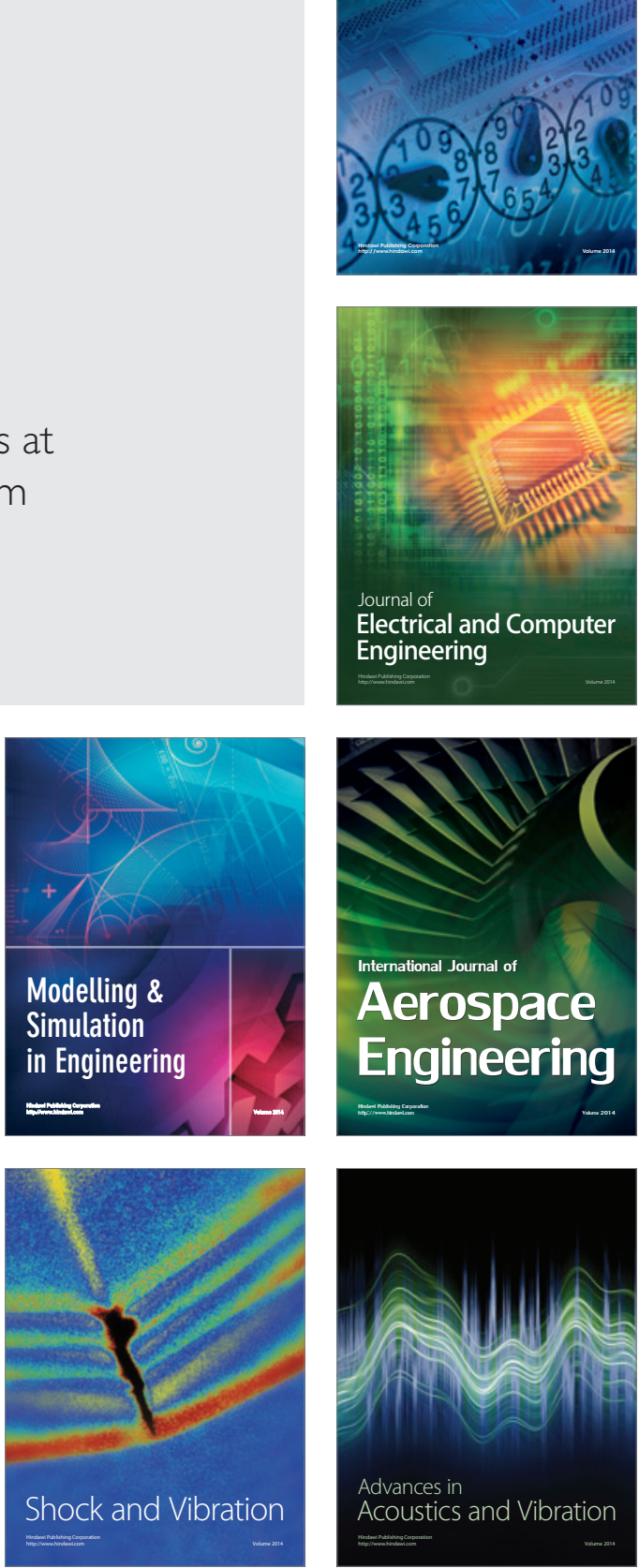